Menlove, H.O., et al. Reproducible Neutron Emission Measurements From Ti Metal in Pressurized D2 Gas. in Anomalous Nuclear Effects in Deuterium/Solid Systems, "AIP Conference Proceedings 228". 1990. Brigham Young Univ., Provo, UT: American Institute of Physics, New York.

\title{
REPRODUCIBLE NEUTRON EMISSION MEASUREMENTS FROM TI METAL IN PRESSURIZED $D_{2}$ GAS
}

\author{
H. O. Menlove, M. A. Paciotti, T. N. Claytor, H. R. Maltrud, \\ O. M. Rivera, and D. G. Tuggle \\ Los Alamos National Laboratory \\ Los Alamos, NM 87545 \\ S. E. Jones \\ Brigham Young University Provo, UT 84602
}

\begin{abstract}
During the past year, we have measured neutron emission from samples of titanium (Ti) metal and sponge in pressurized $\mathrm{D}_{2}$ gas. In January 1990, we improved our sample preparation procedure and our detector sensitivity level so that the neutron-emission measurements are now reproducible, but not yet predictable. We have measured excess neutron emission from the majority of our most recent samples using our high-sensitivity neutron detectors. The improved sensitivity in our new detector system was obtained by using low-radioactive-background stainless steel tubes, a small detector volume with high efficiency, and additional cosmic-ray shielding. Our most sensitive detector consists of two independent segments making up inner and outer rings of $3 \mathrm{He}$ tubes. The combined total efficiency is $44 \%$. In addition to inner and outer ring segments, we have three separate detector systems operating in parallel control experiments to monitor environmental change. We have measured neutron bursts from a variety of samples containing Ti metal and $\mathrm{D}_{2}$ gas. The low-multiplicity bursts, emitting from 2 to $10 \mathrm{n}$, occur much more frequently than the higher multiplicity bursts. By measuring high-mass samples (300 g Ti) over several weeks, with many liquid nitrogen temperature cycles, we have detected neutron emission above the background from most of the samples with a significance level of 3 to $9 \sigma$.
\end{abstract}

\section{INTRODUCTION}

At the time of the workshop on Cold Fusion Phenomena in Santa Fe, New Mexico, May 2325,1989 , we reported on measuring the emission of neutrons from experiments involving titanium (Ti) metal in both pressurized deuterium gas and $\mathrm{D}_{2} \mathrm{O}$ electrolysis type experiments. ${ }^{1}$ Additional data on our gas phase experiments were presented ${ }^{2}$ at the NSF-EPRI workshop, Washington, DC, October 1989.

Early experiments reporting neutron emission have been published by S. E. Jones, ${ }^{3}$ A. Berlin, ${ }^{4}$ F. Scaramuzzi, ${ }^{5}$ V Klyuev, ${ }^{6}$ J. P. K. lyengar, ${ }^{7}$ K. L. Wolf, ${ }^{8}$ and others.

This paper presents an update on our experimental work covering the period through September 1990. Experiments have been performed at our original underground laboratory at Los Alamos as well as at deeper underground tunnels at Los Alamos and near Leadville, Colorado. 
The focus of our work for the past year has been to upgrade the sensitivity and surety of our neutron detectors and to develop sample-preparation procedures to give reproducibility in the neutron emissions.

\section{EXPERIMENTAL UPGRADES}

\section{A. Detectors}

We have designed several neutron detectors for measuring neutrons from cold fusion type experiments. ${ }^{9}$ The high-efficiency detectors are based on ${ }^{3} \mathrm{He}$ gas tubes in a $\mathrm{CH}_{2}$ moderator. The total efficiency of our most advanced detector for $2.3-\mathrm{MeV}\left({ }^{252} \mathrm{Cf}\right)$ neutrons is $44 \%$. The detector shown in Fig. 1 consists of two independent segments making up inner and outer rings of ${ }^{3} \mathrm{He}$ tubes. The inner detector has nine ${ }^{3} \mathrm{He}$ tubes and the outer detector has forty-two ${ }^{3} \mathrm{He}$ tubes. The low-background inner detector has a singles count background of 90 counts/h and a coincidencecount background of only 0.7 counts/h. The corresponding singles efficiency is $19 \%$.

Our initial tubes had aluminium walls for high thermal-neutron counting efficiency; however, for low-background counting, we are now using stainless steel tubes to reduce the radioactive alpha-particle decay background. The background was reduced by about a factor of 5 by changing from aluminum to stainless steel tubes. However, the radioactive decay from the tube walls gives no time-correlated counts, so the aluminum tubes give better neutron-burst detection sensitivity than stainless steel because of their $5 \%$ higher efficiency.

The ${ }^{3} \mathrm{He}$ detector tubes are screwed directly into an aluminum-metal cavity that is sealed to prevent $\mathrm{rf}$ noise penetration. Desiccant is added to keep moisture out of the detector high-voltage area and to prevent any moisture from the test cells from affecting the counting system. Four to six AMPTEK A-III hybrid charge-sensitive preamplifier/discriminators ${ }^{10}$ are inside this sealed cavity. They are placed at the base of the ${ }^{3} \mathrm{He}$ counters to eliminate analog-signal transmission lines that are prone to pick up noise.

Time-correlated neutron counting ${ }^{10}$ is ideal for the neutron-burst results that have been reported. Every neutron count that enters the circuitry triggers the time-correlation counters that check whether there are any other neutron counts within the selected time gate. We use a coincidence time gate of $128 \mu \mathrm{s}$, which corresponds to over three times the neutron die-away time of the detector. If two pulse events are less than $4.5 \mu \mathrm{s}$ apart, they are eliminated from the coincidence gate by a predelay circuit that also eliminates fast coincidence events $(<4 \mu \mathrm{s})$ from electrical noise.

The time-correlated (coincidence) count $R$ is related to the number of neutrons $(N)$ that are detected by the equation

$$
R=\frac{N(N-1)}{2} \text {. }
$$

This relationship assumes that all $N$ neutron events are processed through the $128-\mu$ s coincidence gate in a time less than the gate length. If a burst event plus the thermalization time lasts more than $128 \mu$ s, the value of $N$ will be underestimated. The accidental coincidence counts are measured by sampling the same shift registers after a delay of $1 \mathrm{~ms}$ following each signal pulse.

To process the high throughput of neutrons from a burst in a short time interval, we use up to six amplifier channels with clipping time constants of $180 \mathrm{~ns}$. To reduce deadtime losses, the 
neutron counts are stored in a derandomizing buffer before they enter the shift-register electronics. ${ }^{10}$ Also, all neutron counts trigger the time-correlation circuit without waiting through the gate time.

For low-level neutron counting, it is necessary to distinguish true neutron counts from spurious background noise. Measures to accomplish this include:

1. splitting the $3 \mathrm{He}$ tubes into inside and outside rings and checking that the inside/outside ratio is consistent with neutrons originating from the sample position,

2. splitting the signal output to long $(128 \mu \mathrm{s})$ and short (32 $\mu \mathrm{s})$ gates and checking that the count ratio is consistent with the detector die-away time $(\sim 50 \mu \mathrm{s})$, and

3. adding an external bank of cadmium-covered ${ }^{3} \mathrm{He}$ tubes with no $\mathrm{CH}_{2}$ moderator to count only electrical noise and not neutrons. These external tubes run on the same high-voltage power supply as the primary detector and identify spurious electronic noise events. In practice, the veto events are less frequent than one per week.

The high efficiency of our detector systems results in very good sensitivity for low-level counting. In comparing the sensitivity of different detector designs, a figure of merit is the efficiency $\varepsilon$ squared over the background $\left(\varepsilon^{2} / \mathrm{B}\right)$. The inside ring in the detector system shown in Fig. 1 has an efficiency of $19 \%$ and a background of 90 counts/h resulting in

$$
\frac{\varepsilon^{2}}{B}=\frac{0.0361}{90}=4.0 \times 10^{-4} \mathrm{~h}(\text { singles mode }) .
$$

However, our primary results are for neutron coincidence counting where

$$
\frac{\varepsilon^{2}}{B}=\frac{13 \times 10^{-4}}{0.7}=1.86 \times 10^{-3} \mathrm{~h}(\text { coincidence mode }) \text {. }
$$

In the underground tunnel at Los Alamos, the coincidence background is 0.03 counts/h and this figure of merit improves to

$$
\frac{\varepsilon^{2}}{B}=\frac{13 \times 10^{-4}}{0.03}=4.33 \times 10^{-2} \mathrm{~h}(\text { coincidence mode }) \text {. }
$$

however, this figure of merit is valid only when the background rate dominates the signal. 


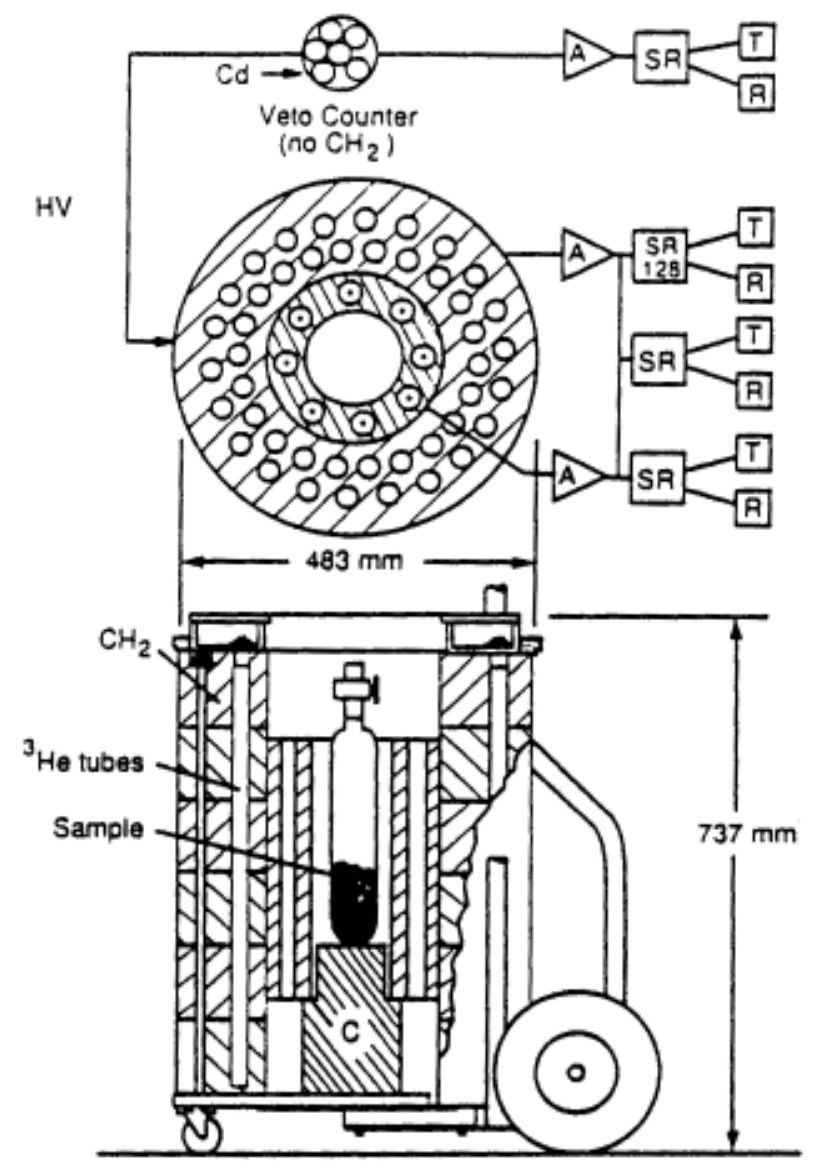

Fig. 1. Schematic diagram of detector system 5 showing the sample, ${ }^{3} \mathrm{He}$ tubes, and the signal processing equipment including the amplifiers (A), shift registers (SR), total scaler $(T)$. and coincidence scaler $(R)$. The veto tubes are used to monitor possible electrical noise.

The coincidence-neutron background decreases by a factor of 70 in going from the ground surface level to our underground laboratory. ${ }^{1}$ The background rate drops by a factor of $2 \times 10^{3}$ in the Los Alamos tunnel and by a factor of $10^{5}$ in the Leadville mine tunnel $1750 \mathrm{ft}$ below the surface (see paper by S. E. Jones, et al., at this meeting).

\section{B. Sample Characteristics and Preparation}

To obtain the reproducible results reported in this paper, we made several improvements in our sample preparation, including:

- $\quad$ large sample cells $(1000 \mathrm{ml})$,

- $\quad$ portable, dual gas cells for adding $\mathrm{D}_{2}$ to the $\mathrm{Ti}$ in the detector,

- $\quad$ larger Ti samples ( 300 g), and

- $\quad$ clean sample preparation and gas loading.

The sample-material summary in Table I also gives the total quantity of gas absorbed by each sample until counting was terminated. The alloys are $\mathrm{Ti}(6 \% \mathrm{Al}, 6 \% \mathrm{~V}$, and $2 \% \mathrm{Sn})$; $\mathrm{Ti}(6 \% \mathrm{Al}$ and $4 \% \mathrm{~V})$; and $\mathrm{Ti}(10 \% \mathrm{~V}, 2 \% \mathrm{Fe}$, and $3 \% \mathrm{Al})$. For sample DD-8, the commercially pure Ti was 
punched from a 3-mm-thick plate, exposing fresh surfaces on the edges.

Motivation for the following procedure comes from five similar samples run in spring 1989, three of which emitted 2-4 bursts of neutrons with multiplicities greater than 20 or $30 .^{1}$

1. Lathe turnings from bars of annealed Ti alloys are cut $0.5 \mathrm{~mm}$ thick and $1.5 \mathrm{~mm}$ wide. The machining is usually done dry. The work hardened turnings are broken into smaller pieces so they can pass through 1-cm-diam opening in the pressure cylinder.

2. Chips are degreased in the sample cylinder with at least three flushes each of reagent grade methylene chloride, methanol, and water. The sample cylinder is agitated by hand for about 5 minutes for each wash. Any hydride powder from the previous experiment is washed out in this step.

3. Connections to the sample cell are either welded or made with high-pressure fittings. The system should be leak-free because the sample pumps itself to a vacuum some time after the $\mathrm{D}_{2}$ gas is introduced. A pressure transducer was used with every sample so that we could measure the gas absorption properties of the titanium. We were able to establish reproducible gas behavior.

4. The water is pumped off, at temperatures up to $120^{\circ} \mathrm{C}$, with clean (oil free) sorption pumps. Heating is done with heat tape on the sample cylinder, the temperature is taken on the outside of the cylinders.

5. Cleaning is completed by purging with purified $\mathrm{D}_{2}$ or $\mathrm{H}_{2}$ at temperatures between $120^{\circ} \mathrm{C}$ and $220^{\circ} \mathrm{C}$. The gas is cleaned with a molecular sieve trap at $75 \mathrm{~K}$. All the connection plumbing from the trap to the sample is heated to $70^{\circ} \mathrm{C}$ so that water vapor is transported out of the system. Purging pressure has varied from 40 to 600 psi and the gas was exhausted to atmosphere back through the trap or into the sorption pumps. The purging cleans the multitude of small cracks that are not accessible by pumping.

6. The temperature is usually increased slowly while purging proceeds, with the objective of reaching at least $160^{\circ} \mathrm{C}$ and about six purge cycles before deuteriding begins. Samples later than DH-14 have been cleaned in helium to reduce the risk of accidental massive deuteriding during the purging. The effect of helium purging on reproducible neutron emissions has not yet been evaluated.

7. Signs of deuteriding sometime show during the hot-purging and cooling process. Starting with sample DD-9, the sample was not allowed to absorb the $\mathrm{D}_{2}$ during purging, and a $\mathrm{D}_{2}$ gas-reserve tank was included for the convenience of adding a measured quantity of $\mathrm{D}_{2}$ to the sample at each LN cycle. Two valves are used between the sample cylinder and the reserve tank with the transducer intermediate so that sample or reserve tank pressure can be monitored. The reserve tank is filled with $30 \mathrm{~L}$ (STP) of purified deuterium before the assembly is transported to the counting area. 


\begin{tabular}{|c|c|c|c|}
\hline Sample & $\begin{array}{l}\text { Ti } \\
\text { Mass (g) }\end{array}$ & Material Composition & Gas absorbed in STD L \\
\hline DD-6 & 200 & Ti sponge & $49 \mathrm{~L} \mathrm{D}_{2}$ \\
\hline DD-7 & 320 & $64 \% \mathrm{Ti}(6,6,2)$ and $\mathrm{Ti}(6,4)$ chips & $51 \mathrm{~L} \mathrm{D}_{2}$ \\
\hline DD-8 & 210 & commercially pure Ti punches and twisted rods & $6.9 \mathrm{~L} \mathrm{D}_{2}$ \\
\hline DD-9 & 280 & $58 \% \mathrm{Ti}(6,6,2)$ and $\mathrm{Ti}(6,4)$ chips & $110 \mathrm{~L} \mathrm{D}_{2}$ \\
\hline DD-10 & 310 & $28 \% \mathrm{Ti}(6,6,2)$ and $\mathrm{Ti}(6,4)$ chips & $124 \mathrm{~L} \mathrm{D}_{2}$ \\
\hline DD-11 & 260 & commercially pure Ti chips & $58 \mathrm{~L} \mathrm{D}_{2}$ \\
\hline DD-12 & 300 & $57 \% \mathrm{Ti}(6,6,2) ; 31 \% \mathrm{Ti}(6,4)$, and $12 \% \mathrm{Ti}(10,2,3)$ & $54 \mathrm{~L} \mathrm{D}_{2}$ \\
\hline DH-13 & 300 & $57 \% \mathrm{Ti}(6,6,2) ; 31 \% \mathrm{Ti}(6,4)$, and $12 \% \mathrm{Ti}(10,2,3)$ & $18 \mathrm{~L} \mathrm{H}_{2} 90 \mathrm{~L} \mathrm{D}_{2}$ \\
\hline DH-14 & 300 & $57 \% \mathrm{Ti}(6,6,2) ; 31 \% \mathrm{Ti}(6,4)$, and 12 "o $\mathrm{Ti}(10,2,3)$ & $15 \mathrm{~L} \mathrm{H}_{2} 90 \mathrm{~L} \mathrm{D}_{2}$ \\
\hline
\end{tabular}

\section{RESULTS}

Most of the neutron-emission results in our recent samples are different from those reported in Ref. 1 in that we have fewer large bursts during the warmup from liquid nitrogen temperature, but we have more small neutron bursts with low multiplicities (2-10 n).

Our normal procedure for the measurements was to cool the sample cell in liquid nitrogen (LN). We then loaded the sample cell with 10-200 psi of $\mathrm{D}_{2}$ gas from the reserve tank. No gas absorption takes place at the $\mathrm{LN}$ temperature $\left(-198^{\circ} \mathrm{C}\right)$; however, during the warmup of the cell in the neutron detector, the Ti slowly starts to absorb the deuterium at about $-100^{\circ} \mathrm{C}$. Normally all of the gas is absorbed by the time the cell reaches room temperature $\left(\sim 22^{\circ} \mathrm{C}\right)$. This procedure was repeated, usually daily, until the 1.4 moles in the $\mathrm{D}_{2}$ gas-reserve tank was absorbed by the sample. The reserve tank was refilled one to four times for samples 9-14 in Table II.

In a few cases, excess singles-mode neutron emission was measured early in the absorption process; however, our detector systems are much more sensitive to time-correlated (coincident) neutron emission because of the lower backgrounds. All of the results reported in this paper correspond to coincidence neutron (burst) counting.

\section{A. Sample Cell DD-6}

The coincidence counting results for sample DD-6 are shown in Fig. 2; each data time interval corresponds to the average coincidence rate for about $24 \mathrm{~h}$ of data collection. In most cases an LN cycle was performed at the start of the counting period. The same procedure was used for the control sample that consisted of the same size steel cylinder filled with $300 \mathrm{~g}$ of Ti chips in $1 \mathrm{~atm}$ of air. In previous experiments, ${ }^{1} \mathrm{H} 2$ control samples had been used, and the results showed no excess neutron emission compared with the steel-plus-air control cylinder background. 


\begin{tabular}{|c|c|c|c|c|}
\hline \multicolumn{5}{|c|}{ Table II. Coincidence Rate Significance Level for Samples in System } \\
\hline \multirow[b]{2}{*}{ Sample } & \multirow[b]{2}{*}{$\begin{array}{l}\text { Measurement } \\
\text { Days }\end{array}$} & \multicolumn{3}{|c|}{ STD Significance Level $(\sigma)$} \\
\hline & & $\begin{array}{l}\text { Av. Of } \\
\text { All Days }\end{array}$ & $\begin{array}{l}1 \text { st } 4 \mathrm{~d} \\
\text { After } \mathrm{D}_{2}\end{array}$ & $\begin{array}{l}\text { Single } \\
\text { High Day }\end{array}$ \\
\hline DD-6 & 7 & 4.7 & 3.0 & 3.4 \\
\hline DD-7 & 11 & 7.4 & 4.9 & 5.5 \\
\hline DD-8 & 19 & 4.5 & 4.0 & 3.6 \\
\hline Ti Control- 1 & 29 & 0 & 1.5 & 2.0 \\
\hline DD-9 & 39 & 5.4 & 3.3 & 9.0 \\
\hline DD-10 & 8 & 0.6 & 2.1 & 2.6 \\
\hline DD-11 & 15 & 2.0 & 2.6 & 3.3 \\
\hline DD-12 & 3 & 0 & 0 & 3.4 \\
\hline DH-13 & 12 & 2.8 & 1.8 & 5.0 \\
\hline DH-14 & 12 & 4.2 & 3.0 & 8.0 \\
\hline Ti Control-2 & 26 & 0 & 1.4 & 2.0 \\
\hline
\end{tabular}

Sample DD-6 was counted in two independent detector systems during the experiment that lasted several weeks. Detector system 5 is shown in Fig. 1, and detector-system 4 is a 16-tube $\left({ }^{3} \mathrm{He}\right)$ inside ring and an 8-tube outside ring as described in Ref. 2. The absolute efficiency for the inside of system 4 is $31 \%$ for $2.3-\mathrm{MeV}$ neutrons $\left({ }^{252} \mathrm{Cf}\right)$.

The significance level of the excess coincidence neutron emission from sample DD-6 was $4.7 \sigma$ in system 4 and $5.0 \sigma$ in system 5 taking into account all of the data runs. Also, the data were evaluated using only the first four days after the initial gas fill and, alternatively, the single high-counting day. All of the data evaluation results for system 4 are presented in Table II and Fig. 3. The comparison of the results from detector system 4 and system 5 is shown in Fig. 4.

Figure 5 shows a summary of the coincidence background measurements with the control sample in the detector (system 4) during the 5-month period covering most of the experiments. Each data interval in Fig. 5 corresponds to the average rate for approximately $24 \mathrm{~h}$ of counting. The average coincidence background from cosmic-ray-induced spallation neutrons increased from 2.62 to 2.91 counts per hour when the gas-reserve tank was included in the detector for samples DD-9 through DD-14. 


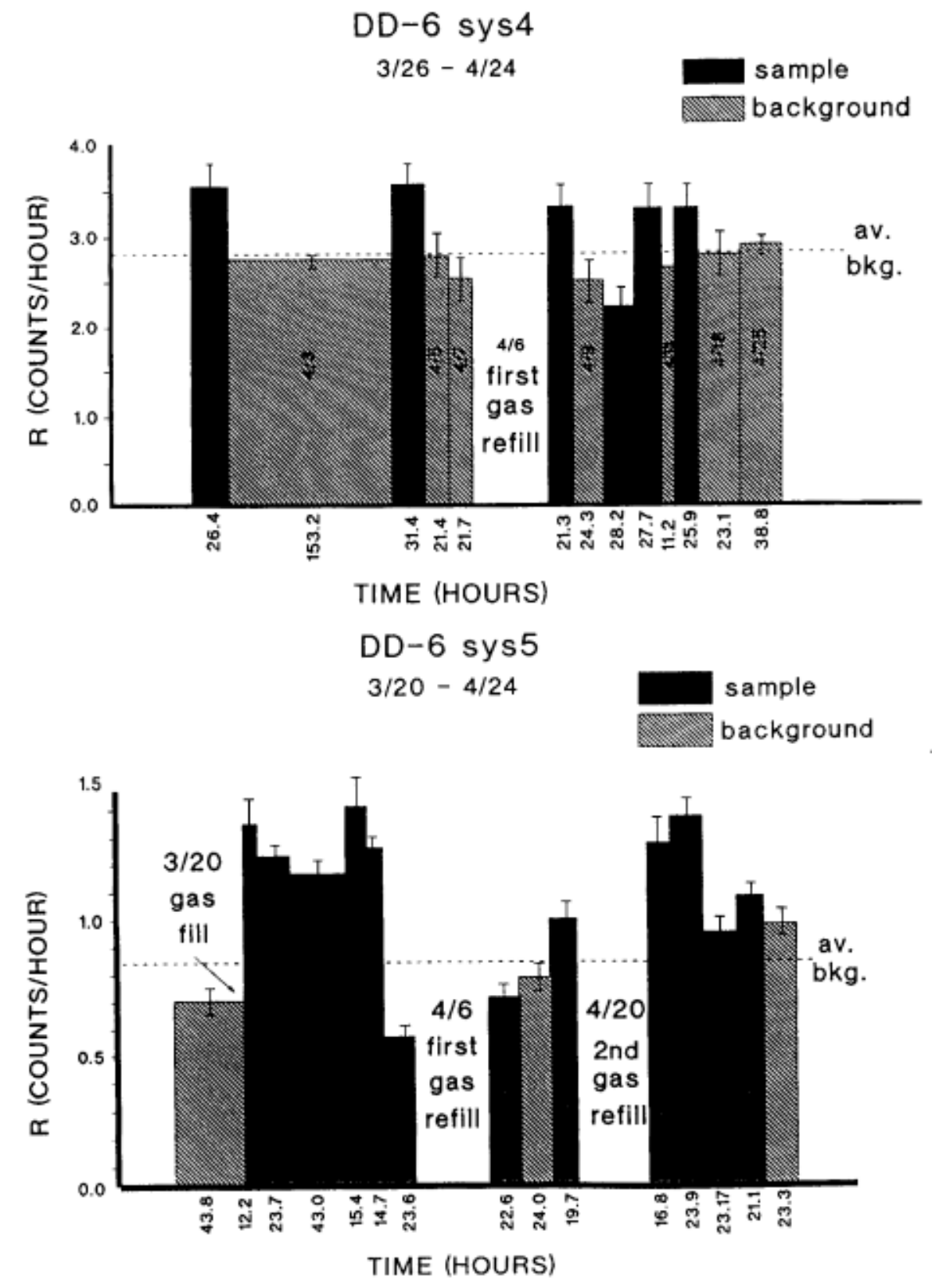

Fig. 2. Coincidence neutron counting results for sample DD-6 where the average neutron counting rate is given in data intervals of typically $24 \mathrm{~h}$. The top data (a) correspond to detector system 4 and the bottom data (b) correspond to detector system 5. 


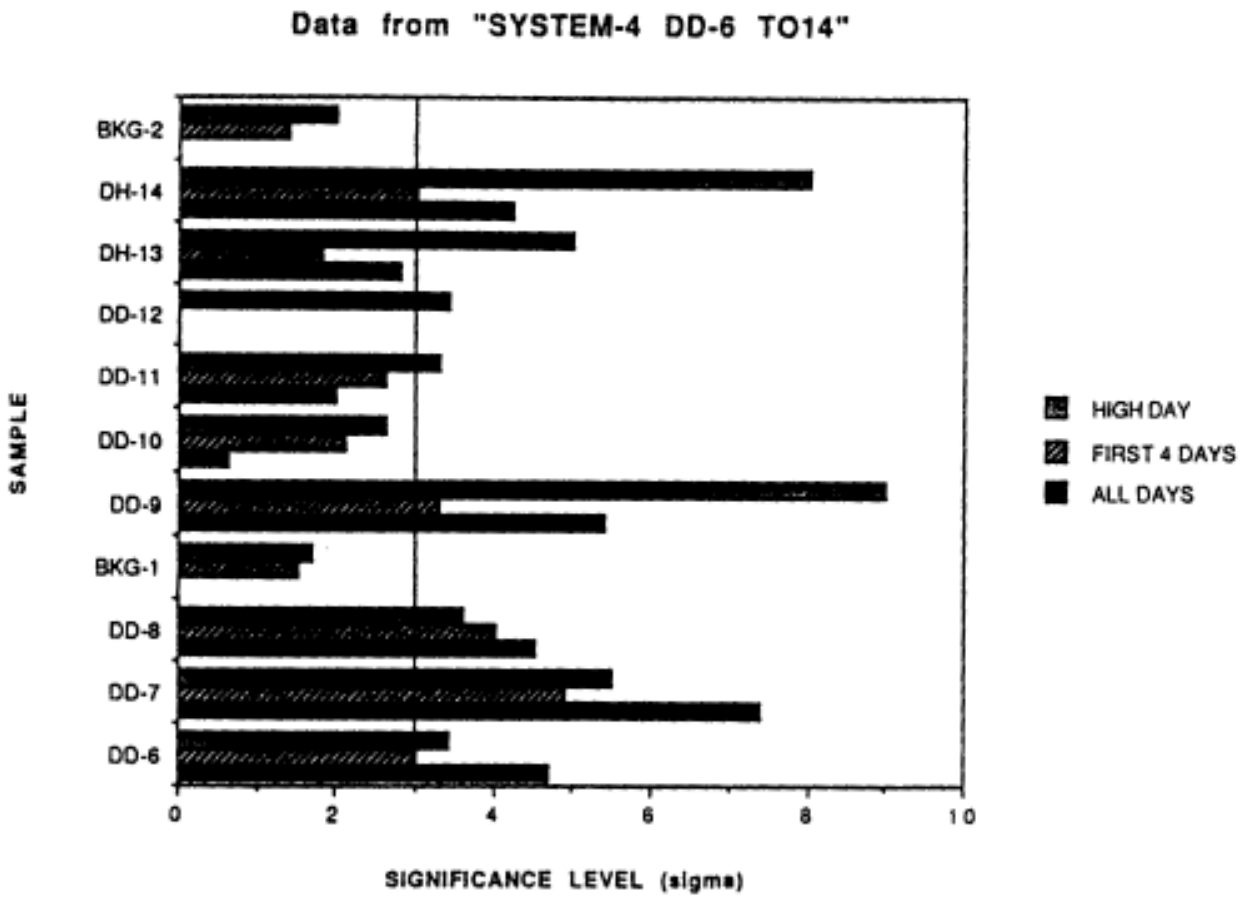

Fig. 3. The significance level in standard deviations for the excess neutron emission from the sample set DD-6 through DH-14. The results are presented for all of the data runs, the first four days after the first $D_{2}$-gas fill, and the single high day. The first background level corresponds to the sample cell without a reserve-gas cell and the second background is for the cell plus the reserve-gas cell.

\section{B. Sample Cell DD-7}

The coincidence counting results for sample DD-7 are shown in Fig. 6 where the top data correspond to detector system 4 and the bottom data correspond to system 5 . The same LN cycle and counting procedure was used for samples DD-7 and DD-6.

When all of the data runs for sample DD-7 are combined, the significance level for the excess neutron emission for system 4 was $7.4 \sigma$ and $8.0 \sigma$ for system 5 . The significance levels, taking the single high-day data for the first four days after the gas fills, are shown in Fig. 3 for system 4.

\section{Sample Cell DD-9}

The coincidence counting results for sample DD-9 are shown in Fig. 7. The same general procedure of LN cycles used for the other samples was used for DD-9. The total counting time including background runs and sample DD- 8 was $\sim 70 \mathrm{~d}$ and there were five $\mathrm{D}_{2}$-gas refills in the reserve tank as shown in Fig. 7. The total amount of $\mathrm{D}_{2}$ gas absorbed was $\sim 110 \mathrm{~L}$. 
ALL RUNS SYS-5 VS. SYS-4

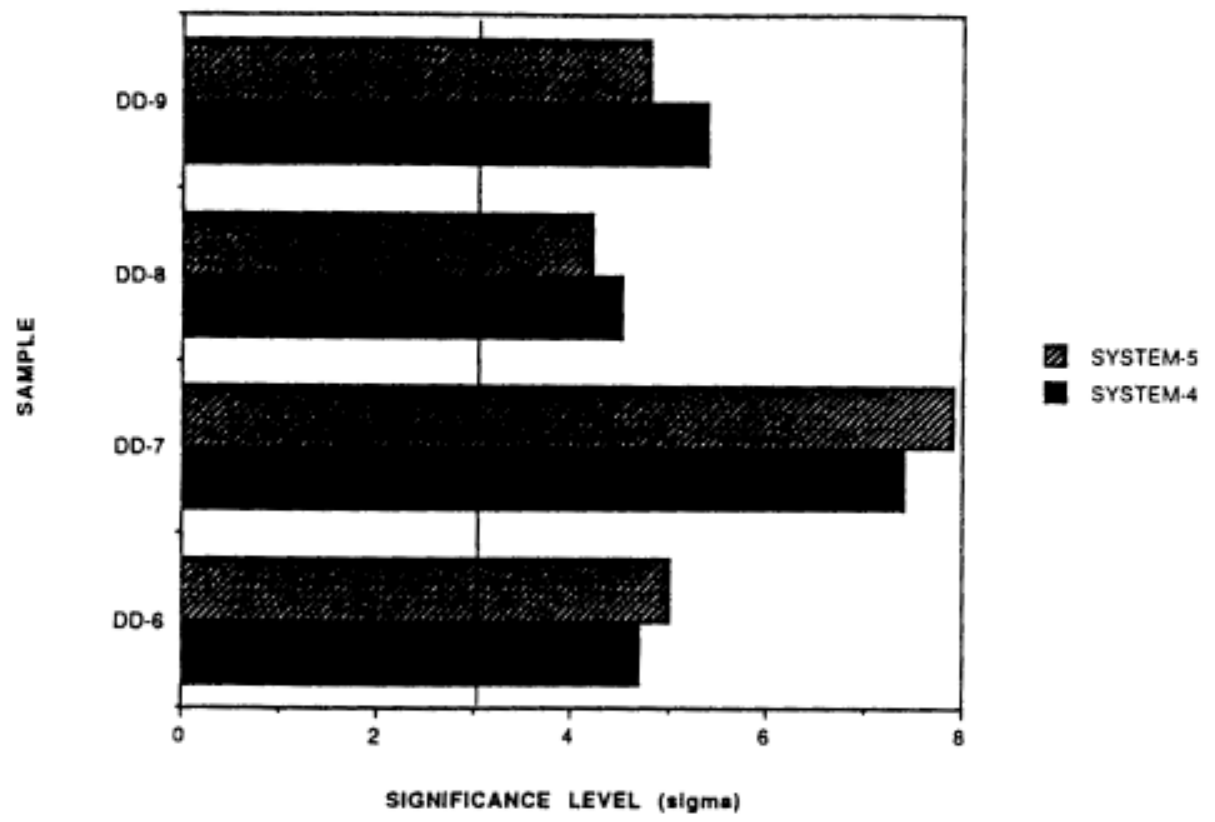

Fig. 4. The comparisons of the significance level of the excess neutron emission (for all days) from systems 4 and 5 is shown for samples DD-6 to DD-9 that were counted in both detector systems.

When all of the data runs were combined, the significance level was $5.4 \sigma$ in system 4 . Figure 3 shows the significance levels for the other data time cuts and Fig. 4 shows the comparison between systems 4 and 5. The significance level is higher in system 4 than in system 5 because most of the data for sample DD-9 were taken on system 4.

\section{DISCUSSION}

While these samples did not produce striking numbers of neutrons or large bursts, some measure of reproducibility was attained. It is possible to repeat the sample preparation and achieve the same gas absorption characteristics. Even though the preparation and $\mathrm{D}_{2}$ gas absorption methods evolved during this series, apparently most of the samples emitted excess neutrons. In contrast, a few very early samples from Ref. 1 contained unique materials, unknown surface contamination, and unknown bottle gas purity but gave some large bursts on successive LN cycles. ${ }^{1}$ Such dramatic results are not easily reproduced. 


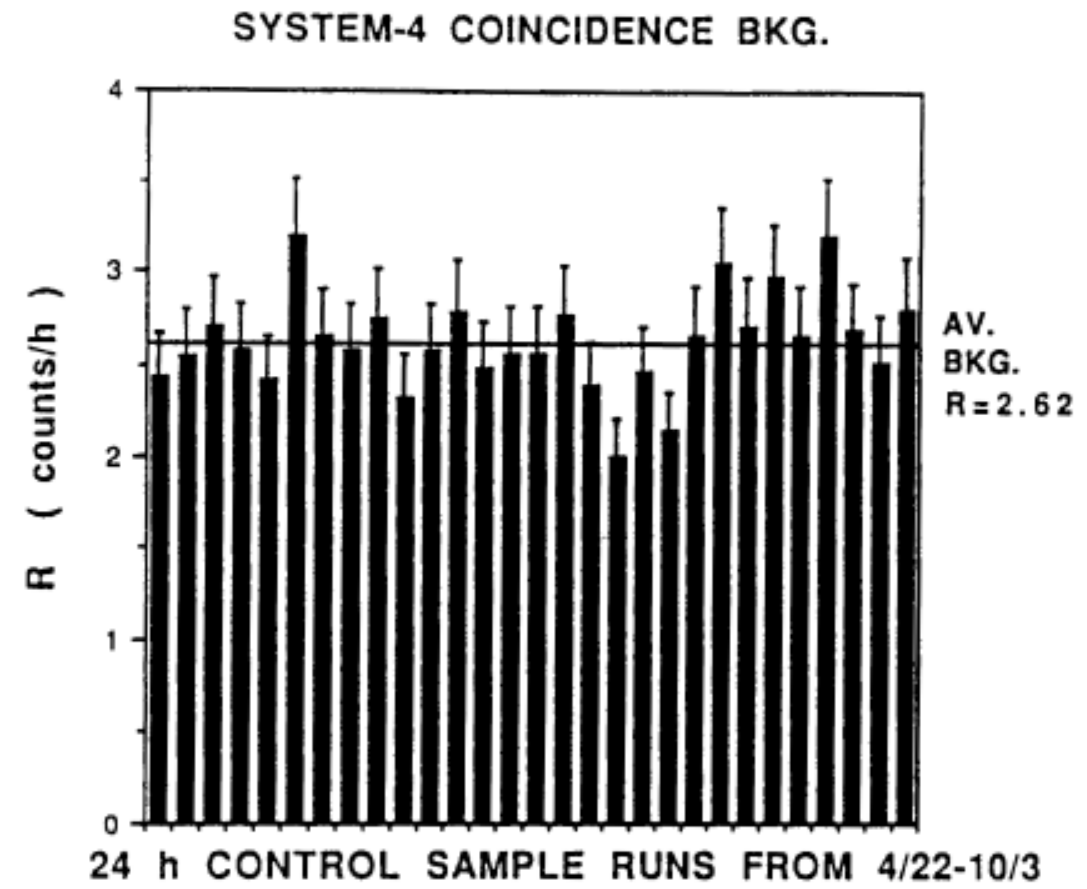

Fig. 5. Neutron coincidence background rate for the control sample without the reserve gas cylinder in detector system 4 where each data interval corresponds to the average rate for $\mathbf{- 2 4} \mathbf{h}$ of counting.

The clean gas and sample preparation limits reoxidation of surfaces that have lost their protective oxide layer by thermal cracking. In this way the sample can absorb $\mathrm{D}_{2}$ at temperatures from $-100^{\circ} \mathrm{C}$ to room temperature during successive $\mathrm{LN}$ cycles until the sample is saturated. The gas uptake behavior suggests the absorption occurs in local regions of the chips rather than uniformly throughout the sample, an effect explained by the heat of reaction concentrating the absorption in one area until near saturation. At the conclusion of each experiment, the chips are all brittle and, without exception, a dull grey color. A small amount of powder will have formed.

We suspect that chips driven to gas saturation do not emit neutrons because neutron emission is normally absent after massive deuteriding. In those instances where neutron emission persists, perhaps the chips peripheral to the hot region have undergone a full spectrum of temperature changes and variable absorption. Our procedure allows a large fraction of the chip population to experience partial hydriding.

Gas absorption always increases after the first cycle from $75 \mathrm{~K}$ to room temperature, and the $\mathrm{D}_{2}$ quantity is limited to avoid excess temperature rise. With the reserve tank, the gas quantity per cycle becomes a controllable parameter and will be explored more in the next stage of experiments. 

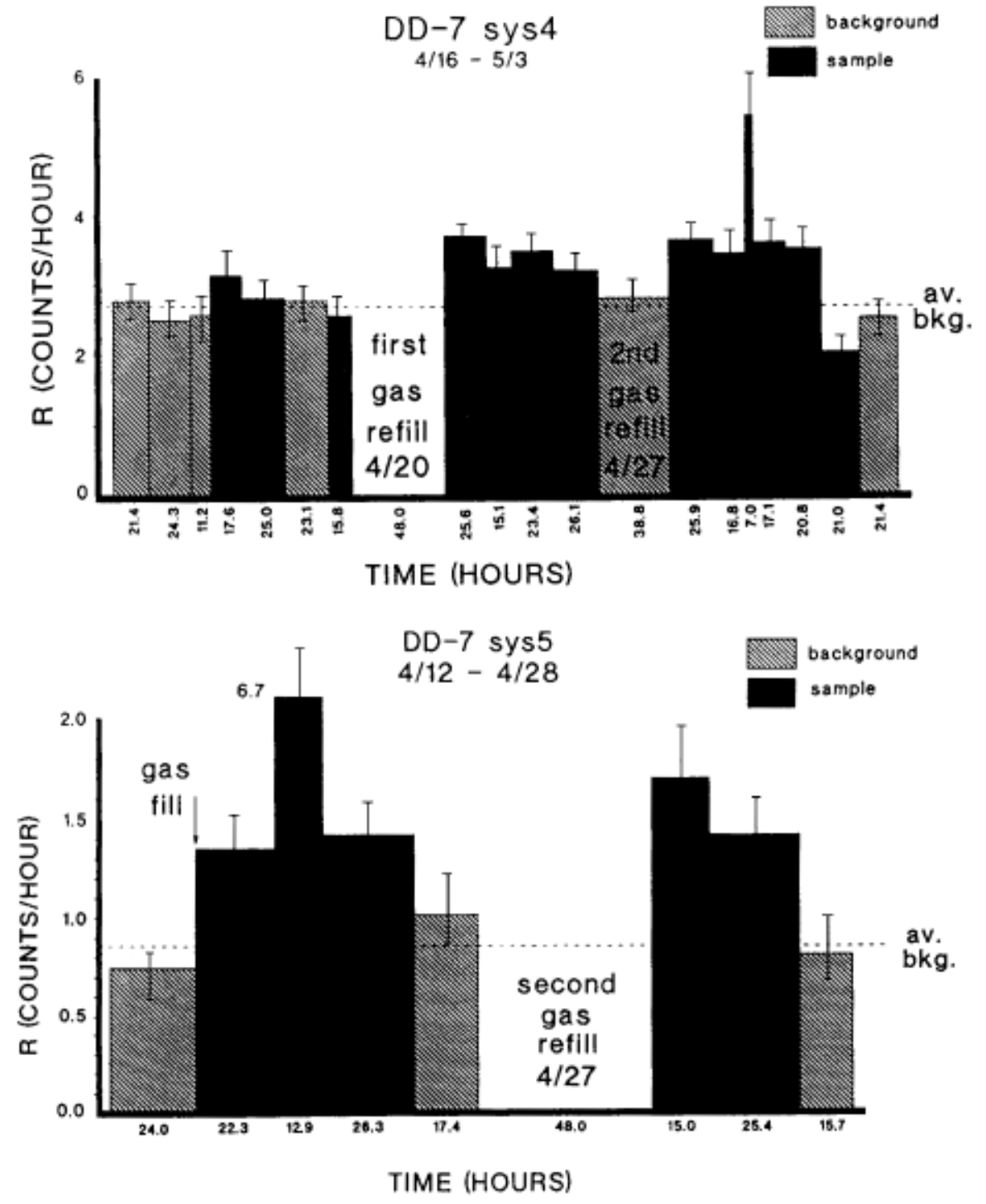

Fig. 6. Coincidence neutron counting results for sample DD-7 where the aver- age neutron counting rate is given in data intervals of typically $24 \mathrm{~h}$. The top data (a) correspond to detector system 4 and the bottom data (b) correspond to detector system 5. 


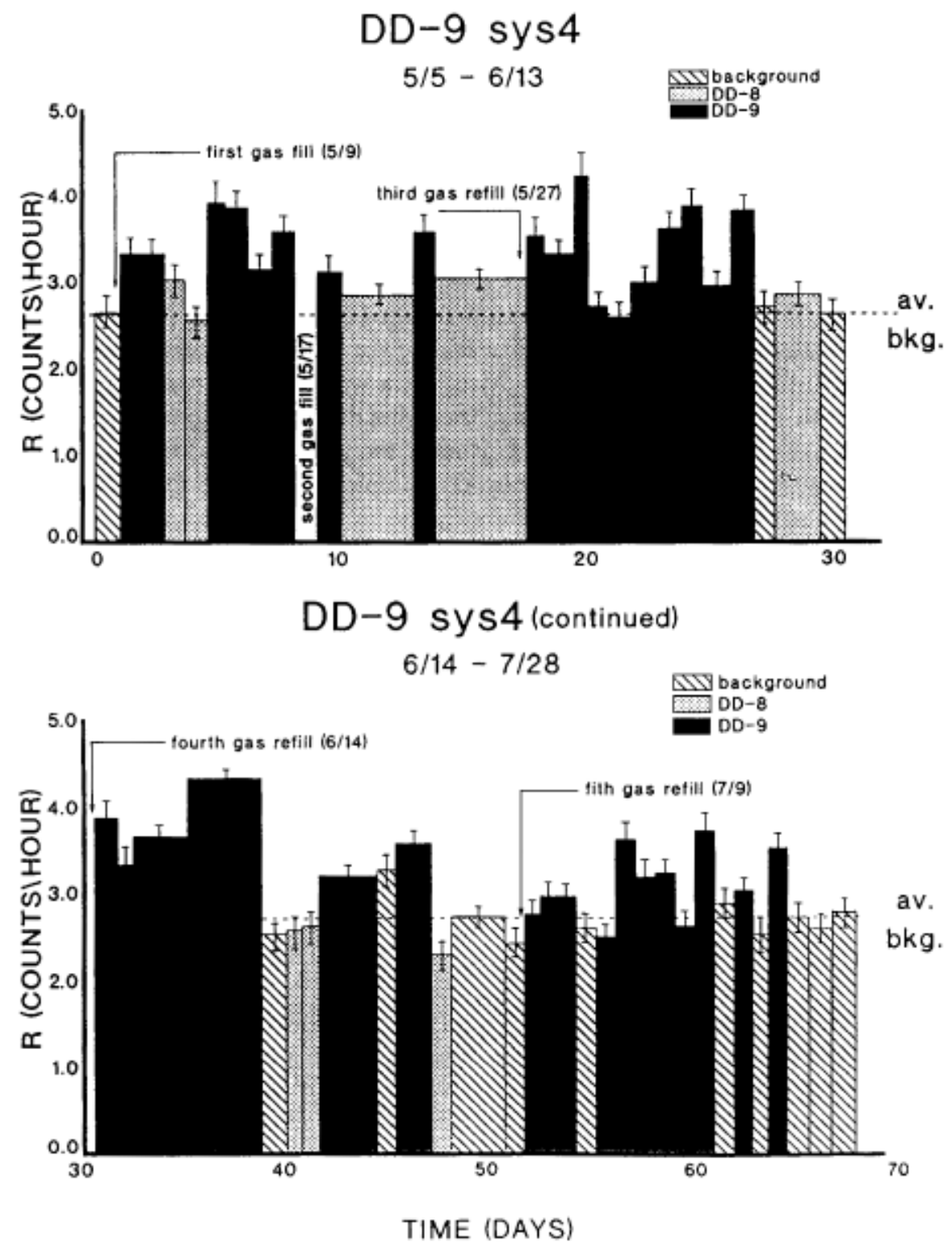

Fig. 7. Coincidence neutron counting results for sample DD-9 in system 4 where the neutron counting rates are averaged over data intervals of typically $24 \mathrm{~h}$. Also shown are the counting results from sample DD-8 and the dummy sample during the same time period.

\section{SUMMARY}

The majority of the coincidence counts giving the results shown in Figs. 2 through 7 have small multiplicities resulting in small values of $R$ ( 1 to 3 counts). The $4.5 \mu$ s predelay in the timecorrelation electronics effectively eliminates electronic noise for the repetitive small coincidence events. For example, to give $R=1$, the electronic noise oscillation would have to have an initial trigger followed by oscillations with one and only one oscillation above the threshold after $4.5 \mu$ s. Experimental tests have verified that electrical noise sources do not give repetitive small values of $R$. 
The clean Ti sample and gas preparation resulted in rapid (a few minutes) $\mathrm{D}_{2}$ gas absorption at room temperature. The high level of deuteriding the Ti chips seemed to be related to the small multiplicities in the neutron emissions. Also, there was not the strong correlation between the neutron emission and the $-30^{\circ} \mathrm{C}$ temperature transition during warmup from $\mathrm{LN}$ using these procedures that we have previously observed. ${ }^{1}$ Most of the samples listed in Table I gave excess neutron emission at the 3 to $9 \sigma$ significance level. Samples DD-10 and DD-12 did not yield significant excess neutron emission. The uncertainty in the background was included in the analysis.

The neutron emission is not likely originating from other reactions in the deuterium, such as photodisintegration, because the emission persists in the low-background underground locations, and also the deuterium inventory increases as the experiment progresses because of the multiple gas refills; however, the neutron-emission rate decreases. At the end of the experiment, the deuterium inventory is at a maximum and the neutron-emission rate has usually returned to the background level.

Recently, we measured additional Ti samples in pressurized $\mathrm{D}_{2}$ gas in an underground tunnel at Los Alamos. We have seen large burst events (100-900 $\mathrm{n}$ ) in this shielded location with a correlation to the temperature warmup from the LN cycles. Also, large burst events have given the expected ratios of counts in the inner and outer tube segments of detector systems 4 and 5 . The evaluation of this data is in progress, and the results will be reported in the future. 


\section{REFERENCES}

1. H. O. Menlove, M. M. Fowler, E. Garcia, M. C. Miller, M. A. Paciotti, R. R. Ryan, and S. E. Jones, "Measurements of Neutron Emission from Ti and Pd in Pressurized $\mathrm{D}_{2}$ Gas and $\mathrm{D}_{2} \mathrm{O}$ Electrolysis Cells," Journal of Fusion Energy, 9(4) (1990).

2. H. O. Menlove, E. Garcia, and S. E. Jones, "Update on the Measurements of Neutron Emission from Ti Samples in Pressurized $\mathrm{D}_{2}$ Gas," Proceedings of NSF-EPRI Workshop, Washington, DC, Oct. 16-18, 1989 (to be published).

3. S. E. Jones, et al. Nature 338, 737-740 (April 27, 1989).

4. A. Bertin, et al., Nuovo Cimento 101A, 997-1004 (June 1989).

5. F. Scaramuzzi, et al., Frascati Research Center report, April 18, 1989.

6. V. A. Klyuev, et al., Soviet Techniques of Physics Letters, 12, 551 (1986).

7. P. K. Kyengar, et al., Fusion Technology 18(1), 32-94 (August 1990), (also published as BARC-1500)

8. K. L. Wolf, "Neutron Emission and the Tritium Content Associated with Deuterium Loaded Palladium and Titanium Metals," Highlights of papers presented at the Workshop on Cold Fusion Phenomena, Santa Fe, New Mexico, May 23-25 (1989), p. 2.

9. H. O. Menlove and M. C. Miller, "Neutron-Burst Detectors for Cold-Fusion Experiments," Nucl. Instr. Meth. in Physics Research, to be published (1990).

10. J. E. Swansen, P. R. Collinsworth, and M. S. Krick, Los Alamos Scientific Laboratory report LA-8319-MS (April 1980). Also published in Nucl. Instr. Meth., 176, 555. 